\title{
The Effect of Surface Functionalization on the Immobilization of Gold Nanoparticles on Graphene Sheets
}

\author{
Min Song, Juan Xu, and Changzi Wu \\ Key Laboratory of Energy Thermal Conversion and Control of Ministry of Education, School of Energy and Environment, \\ Southeast University, Nanjing 210096, China \\ Correspondence should be addressed to Min Song, sm@seu.edu.cn
}

Received 17 February 2012; Revised 26 March 2012; Accepted 28 March 2012

Academic Editor: Kun-Lin Yang

Copyright ( $(2012$ Min Song et al. This is an open access article distributed under the Creative Commons Attribution License, which permits unrestricted use, distribution, and reproduction in any medium, provided the original work is properly cited.

In our study, graphene oxide is synthesized by Hummers method. And then, carboxylic acid functionalized graphene (graphene$\mathrm{COOH}$ ), thiol-functionalized graphene (graphene-SH), and highly dispersive graphene are prepared by chemical modification of respective groups on the graphene surface. Furthermore, we explore a solution-based approach to prepare three differently functionalized graphene-gold composites by one-step chemical reduction of $\mathrm{AuCl}_{4}{ }^{-}$ions in respective functionalized graphene suspensions, where the gold nanoparticles are deposited on the functionalized graphene surface during their synthesis process. In addition, we compare the influence of surface functionalization on the growth of gold nanoparticles on graphene surface. Transmission electron morphology (TEM) and ultraviolet-visible (UV-Vis) spectroscopy are employed to study the effect of surface functionalities on AuNPs distribution onto the graphene surface and demonstrate the successful immobilization of AuNPs on graphene surface.

\section{Introduction}

The increasing interest in carbon family materials has opened new ways for producing arrays of novel functional nanomaterials. Among them, graphene, one-atom thick planar sheet of hexagonally arrayed sp2 carbon atoms, has attracted tremendous scientific attention in recent years. Graphite oxide (GO) is prepared from graphite by chemical oxidation, and then converted into a graphene nanosheets in bulk quantity by chemical and thermal reduction in solution to produce graphene [1-4]. Since abundant oxygen functional groups on the both basal plane and edge of GO can be used as anchoring sites for metal nanoparticles, it is possible to use them as a support to produce graphene-nanoparticle hybrids [5]. Furthermore, the covalently attaching oxygen groups, including hydroxyl, epoxy groups on the basal plane and carboxylic groups at the edge of GO surface is also versatile and effective method to prepare graphene-based hybrid materials by the chemical modification of graphene [6].
The dispersion of metal nanoparticles on graphene sheets may result in the production of some excellent materials for various potential applications [7-10]. Among noble nanoparticles, AuNPs are one of the most studied nanomaterials, due to their remarkable properties [11-14]. A number of studies show that the hybridization of gold nanoparticles with GO followed by chemical reduction could improve the electrical conductivity and Raman signal [15, 16]. The hybrid materials prepared through the various functionalization strategies show potential applications in many fields such as catalyst, electronics, and biology [17-23]. However, the drawbacks for graphene-based hybrid materials are that the particle diameters of prepared gold nanoparticles are nonuniform, and the interaction between GO and AuNPs are weak.

Therefore, we explore a simple approach to prepare three differently functional graphene materials as substrates to fabricate novel nanohybrid materials. The interaction between thiol, carboxyl, and oxygen groups and AuNPs is compared in the paper. The thiol-functionalized graphene 


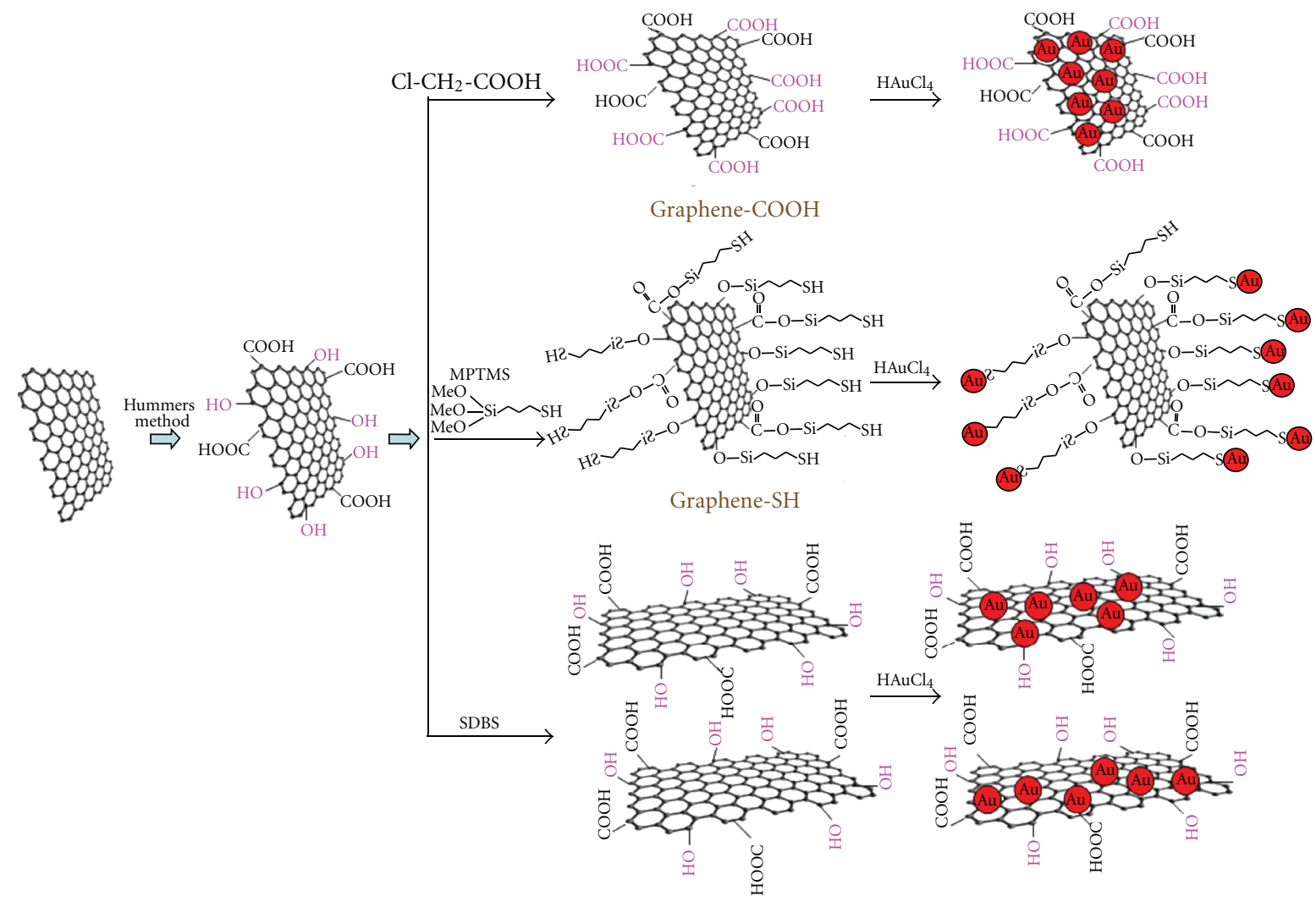

FIGURE 1: Schematic illustration on the preparation route to immobilize gold nanoparticles onto differently functionalized graphene surface.

(graphene-SH)/Au composites show the best performance compared to the other two hybrid materials due to the strong interaction between $-\mathrm{SH}$ and $\mathrm{Au}$, offering an effective way to overcome the drawbacks mentioned above.

\section{Material and Methods}

2.1. Preparation of Differently Functionalized Graphene. The natural graphite was bought from Sigma. The graphite oxide (GO) was prepared from purified natural graphite according to Hummeras's method.

Carboxylic acid functionalized graphene (graphene$\mathrm{COOH})$ produced as follows. $\mathrm{GO}$ aqueous suspension $(5 \mathrm{~mL})$ was diluted as to give a concentration of $2 \mathrm{mg} / \mathrm{mL}$, and then bath-sonicated for $1 \mathrm{~h}$ to give a clear solution. $\mathrm{NaOH}(1.2 \mathrm{~g})$ and chloroacetic acid $\left(\mathrm{Cl}-\mathrm{CH}_{2}-\mathrm{COOH}\right)$ $(1.0 \mathrm{~g})$ were added to the GO suspension and bath-sonicated for $1-3 \mathrm{~h}$ to convert the $-\mathrm{OH}$ groups into $-\mathrm{COOH}$. The resulting $\mathrm{GO}-\mathrm{COOH}$ solution was neutralized and purified by repeated rinsing and filtrations.

Thiol-functionalized graphene (graphene-SH) was produced as follows. The synthesized graphite oxide was suspended in methylene dichloride by sonication for $10 \mathrm{~min}$. And then, (3-mercaptopropyl) trimethoxysilane (MPTMS) was added into $2 \mathrm{mg} / \mathrm{mL}$ GO suspension by stirring-reflux method under nitrogen atmosphere. The filtrated solid was washed with tetrahydrofuran and ethanol three times to remove organic matter and dried in a vacuum oven at $60^{\circ} \mathrm{C}$ for $4 \mathrm{~h}$.

Highly dispersive graphene were produced as follows. The synthesized graphite oxide was suspended in water by sonication for $1 \mathrm{~h}$. The suspension was mixed with sodium borohydride and sodium dodecyl benzene sulfonate by refluence at $80^{\circ} \mathrm{C}$ for $16 \mathrm{~h}$. And then, the product was achieved by centrifugation and washing with water.

\subsection{Preparation of Differently Functionalized Graphene-Gold} Composites. The $2 \mathrm{mg}$ graphene- $\mathrm{COOH}$, graphene- $\mathrm{SH}$, and dispersive graphene were, respectively, suspended in $1 \mathrm{wt} \%$ $\mathrm{HAuCl}_{4}$ solution $(2 \mathrm{~mL})$ by sonication for $5 \mathrm{~min}$ to make relative materials dispersed equably. The suspended solution was then diluted to $200 \mathrm{~mL}$ with doubly distilled water and heated to boiling while stirring. Afterward, $20 \mathrm{~mL}$ sodium citrates were added to the boiling solution which was kept heating for $30 \mathrm{~min}$ with magnetic stirring until the color of the solutions did not change. Then, the sample was cooled to room temperature, then separated in the centrifuge and washed with doubly distilled water five times. The resulting products were dried in a vacuum oven at $80^{\circ} \mathrm{C}$ for $12 \mathrm{~h}$.

2.3. Characterization. TEM images were obtained by using JEM2000FX (JEOL). UV-Vis absorption spectroscopy was performed on Hitachi U-4100. 

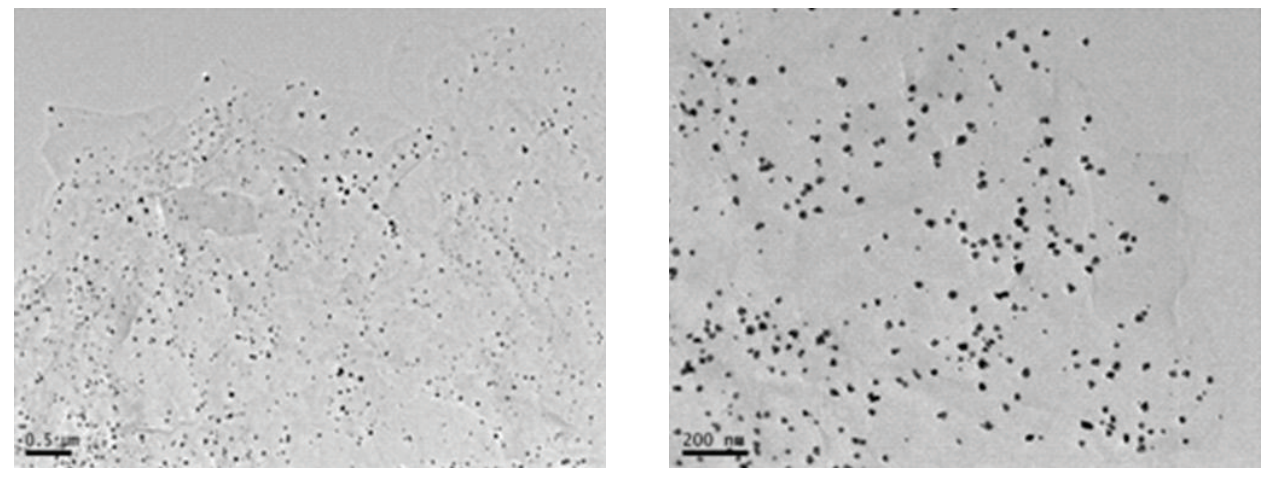

(a)
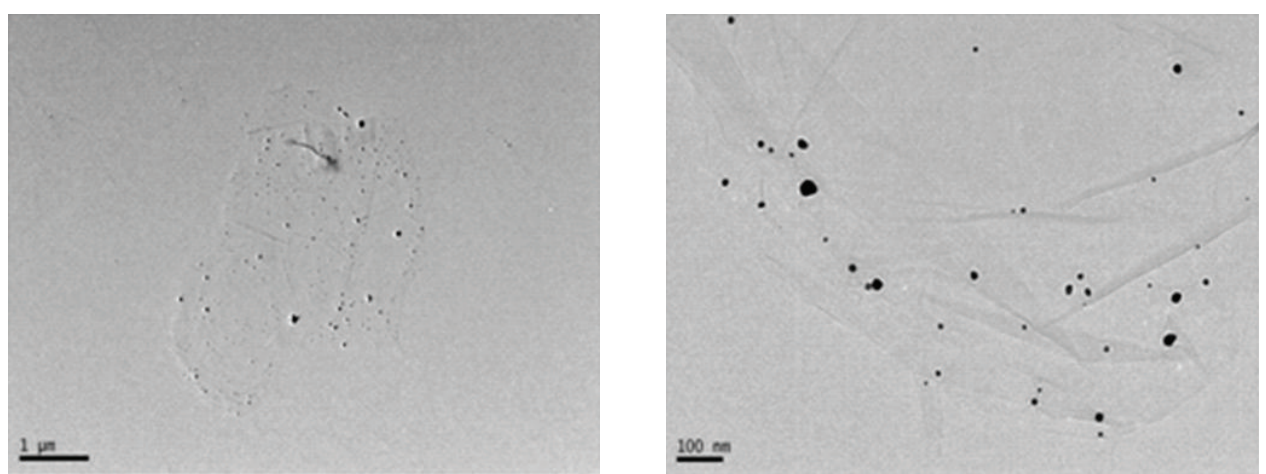

(b)
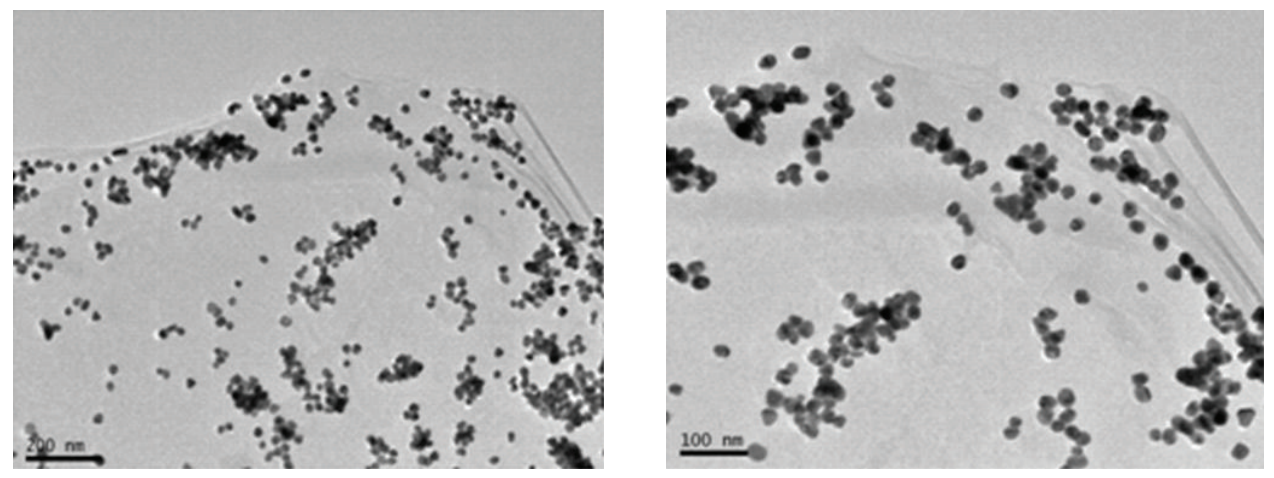

(c)

Figure 2: TEM images of highly dispersive graphene/Au (a), carboxylic acid functionalized graphene /Au (b), and thiol-functionalized graphene/Au hybrids (c).

\section{Results and Discussion}

The graphene oxide sheet, the oxidation of a graphene sheet, can be easily obtained by chemical conversion of inexpensive graphite oxide. Graphene oxide can be used as substrate for deposition of metal nanoparticles due to their unique advantages. For example, graphene oxide sheets have large surface areas and contain the abundance of functional groups (such as carboxyl, carbonyl, hydroxyl, and epoxide) on the surfaces and edges, which facilitate the synthesis of hybrids. Therefore, we firstly use the graphene oxide sheet as the substrate to synthesize carboxylic acid functionalized graphene (graphene-COOH), thiol-functionalized graphene
(graphene-SH), and highly dispersive graphene, respectively. And then, the functional graphenes are immersed in $\mathrm{HAuCl}_{4}$ solution by one step chemical reduction to form the functional graphene-Au hybrids. Figure 1 illustrates the synthetic route for immobilization of Au nanoparticles onto the three differently functional graphene surfaces to obtain novel hybrids.

Morphologies of Au nanoparticles deposited on functional graphene sheets have been characterized by TEM. Figure 2 shows the typical TEM images of as-synthesized hybrids. As for dispersive graphene/Au hybrids, gold nanoparticles appear as dark dots with an average particle size of $20 \mathrm{~nm}$. It is clearly seen in Figure 2(a) that the 


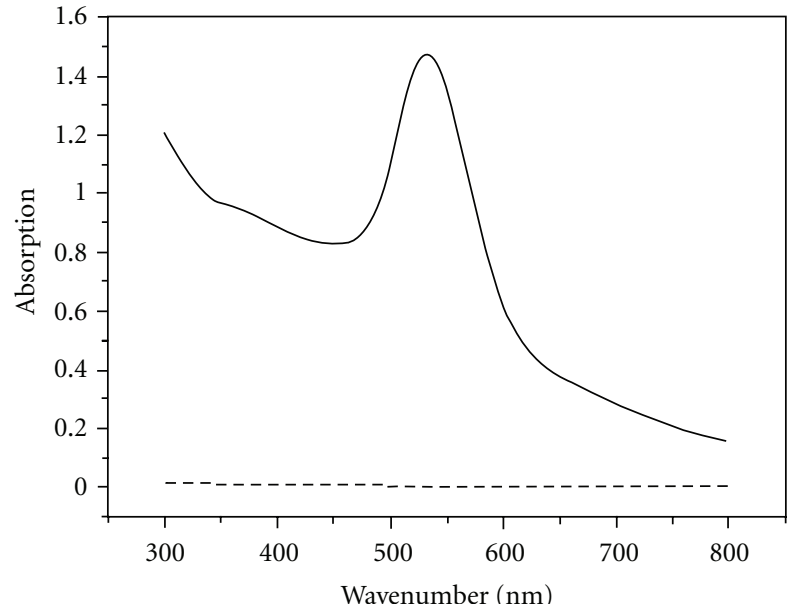

(a)

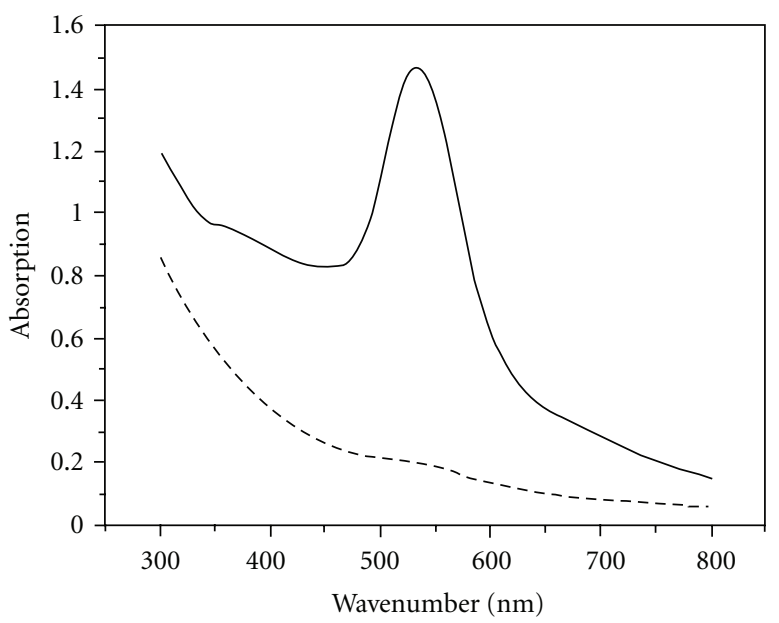

(b)

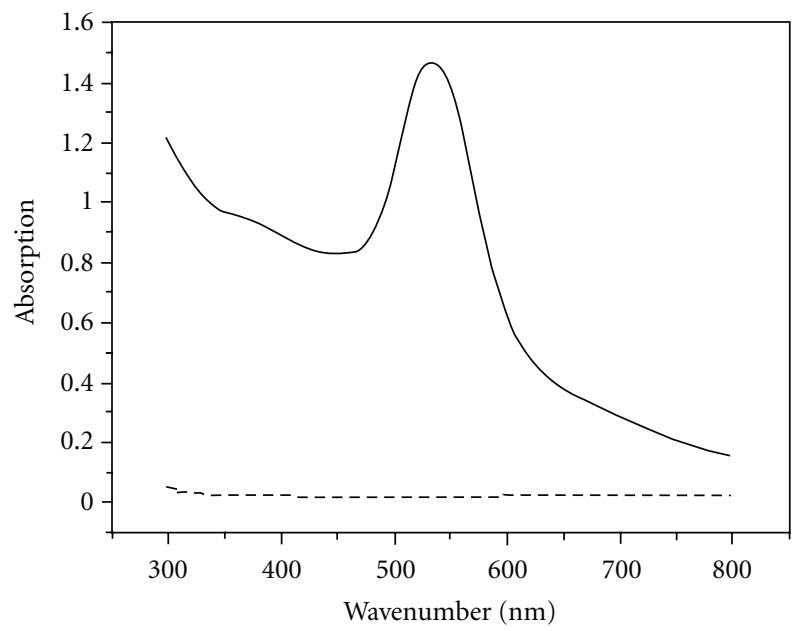

$-\mathrm{Au}$

- - Graphene-SH/Au

(c)

Figure 3: UV-vis absorption spectra of colloid gold solution and centrifugal suspension in the presence of highly dispersive graphene/Au (a), carboxylic acid functionalized graphene / Au (b), and thiol-functionalized graphene/Au hybrids (c). dispersive graphene oxide surface is uniformly decorated by the Au nanoparticles with very few aggregations, indicating a strong interaction between the dispersive graphene oxide and Au nanoparticles. The possible mechanism is that the abundant oxygen functional group on the graphene oxide surface might be used as nucleation sites for growth of $\mathrm{Au}$ nanoparticles. Meanwhile, much quantity of small aromatic conjugated domains on graphene oxide sheet will act as an electron-donating source to reduce $\mathrm{Au}$ ions and form smaller Au nanoparticles [24-26].

However, as for the graphene- $\mathrm{COOH} / \mathrm{Au}$ in Figure 2(b), the sizes of the Au nanoparticles (black dots) are in the range of $8-40 \mathrm{~nm}$. And they occupy only a very small portion of the carboxylic group functional graphene sheet surface. This is reasonable because GO is converted into graphene$\mathrm{COOH}$, much more oxygen containing groups were changed to carboxylic acid groups. Therefore, fewer functional groups will remain as nucleation sites, resulting in the formation of fewer particles.

In addition, we prepare thiol group functionalized graphene with a simple and effective method. And then, the thiol groups are surrounded by $\mathrm{AuCl}_{4}{ }^{-}$that turned into small gold nanoparticles when meeting the reduce agents. Simultaneously, thiol groups could strongly bind gold nanoparticles. The success of functionalization reaction could be demonstrated from the TEM image in Figure 2(c). The results illustrate that the size and shape of Au nanoparticles are much more uniform compared to those on the other two hybrids. Au nanoparticles are distributed on the whole surface of thiol group functional graphene sheet. However, there is a little agglomeration on the surface. This is possibly due to that the graphene has more thiol functional groups, which provided stronger driving force for self-assembly of $\mathrm{Au}$ nanoparticles (GNPs).

In addition, to furthermore demonstrate the success functionalization, we have further investigated the amount of the synthesized gold nanoparticles on the three differently functional graphene by using UV-vis absorption spectroscopy to explore the efficiency of the respective synthesis. As shown in Figure 3, the absorption peak of $\mathrm{Au}$ nanoparticles are observed at $520 \mathrm{~nm}$. After one step chemical reduction of Au ions in three differently functional graphene, the UV-Vis absorption spectroscopy of respective centrifugal suspensions show no absorption peak at $520 \mathrm{~nm}$. The results indicate that the $\mathrm{Au}$ nanoparticles have been coated on the functional graphene surface, therefore, no gold absorption peak is observed. Therefore, the results of our UV-vis absorption spectroscopic studies of gold nanoparticles synthesized and the respective centrifugal suspensions in the presence of functional graphene indicate that more than $99 \%$ of the synthesized gold nanoparticles have been coated on the three differently functional graphene surface.

\section{Conclusion}

We prepare three functionalized graphene by a simple and effective method. Further, a straightforward one-step chemical synthesis method of Au nanoparticles on functionalized 
graphene surfaces is proposed. The results illustrate that the dispersive GO surface are uniformly decorated by the Au nanoparticles with very few aggregations for dispersive graphene/Au hybrids. And, the sizes of the Au nanoparticles show the wide range and occupy only a very small portion of the carboxylic group functional graphene sheet surface for graphene- $\mathrm{COOH} / \mathrm{Au}$ hybrids. The results illustrate that the size and shape of Au nanoparticles are much more uniform compared to those on the other two hybrids due to the strong interaction between thiol groups and gold nanoparticles.

\section{Acknowledgments}

The authors are grateful for the National Natural Science Foundation of China (20907008), the financial support of the National Hi-tech Research and Development Program of China (973) (2010CB732206 and 2011CB201505), and Specialized Research Fund for the Doctoral Program of Higher Education (20090092120010). Dr. Song specially thanks the support of the Foundation for Southeast university Excellent Young Teacher.

\section{References}

[1] D. Li, M. B. Muller, S. Gilje, R. B. Kaner, and G. G. Wallace, "Processable aqueous dispersions of graphene nanosheets," Nature Nanotechnology, vol. 3, no. 2, pp. 101-105, 2008.

[2] S. Park, J. H. An, R. D. Piner et al., "Aqueous suspension and characterization of chemically modified graphene sheets," Chemistry of Materials, vol. 20, no. 21, pp. 6592-6594, 2008.

[3] X. B. Fan, W. C. Peng, Y. Li et al., "Deoxygenation of exfoliated graphite oxide under alkaline conditions: a green route to graphene preparation," Advanced Materials, vol. 20, no. 23, pp. 4490-4493, 2008.

[4] S. Park, J. H. An, I. Jung et al., "Colloidal suspensions of highly reduced graphene oxide in a wide variety of organic solvents," Nano Letters, vol. 9, no. 4, pp. 1593-1597, 2009.

[5] C. C. Chien and K. T. Jeng, "Effective preparation of carbon nanotube-supported Pt-Ru electrocatalysts," Materials Chemistry and Physics, vol. 99, no. 1, pp. 80-87, 2006.

[6] Y. Li, W. Gao, L. Ci, C. Wang, and P. M. Ajayan, "Catalytic performance of Pt nanoparticles on reduced graphene oxide for methanol electro-oxidation," Carbon, vol. 48, no. 4, pp. 1124-1130, 2010.

[7] R. Patakfalvi, D. Diaz, P. Santiago-Jacinto, G. RodriguezGattorno, and R. Sato-Berru, "Anchoring of silver nanoparticles on graphite and isomorphous lattices," Journal of Physical Chemistry C, vol. 111, no. 14, pp. 5331-5336, 2007.

[8] M. Shirai, K. Igeta, and M. Arai, "Formation of platinum nanosheets between graphite layers," Chemical Communications, no. 7, pp. 623-624, 2000.

[9] S. K. Shaikhutdinov and F. J. C. Santos Aires, "Evolution of the rhodium colloid supported on graphite studied by atomic force microscopy in the tapping mode," Langmuir, vol. 14, no. 13, pp. 3501-3505, 1998.

[10] X. J. Yang, Y. Makita, Z. H. Liu, and K. Ooi, "Novel synthesis of layered graphite oxide-birnessite manganese oxide nanocomposite," Chemistry of Materials, vol. 15, no. 6, pp. 1228-1231, 2003.

[11] Z. Zhong, S. Patskovskyy, P. Bouvrette, J. H. T. Luong, and A. Gedanken, "The surface chemistry of Au colloids and their interactions with functional amino acids," Journal of Physical Chemistry B, vol. 108, no. 13, pp. 4046-4052, 2004.

[12] J. Zhou, J. Ralston, R. Sedev, and D. A. Beattie, "Functionalized gold nanoparticles: synthesis, structure and colloid stability," Journal of Colloid and Interface Science, vol. 331, no. 2, pp. 251262, 2009.

[13] J. Han, Y. Liu, and R. Guo, "Facile synthesis of highly stable gold nanoparticles and their unexpected excellent catalytic activity for suzuki-miyaura cross-coupling reaction in water," Journal of the American Chemical Society, vol. 131, no. 6, pp. 2060-2061, 2009.

[14] A. P. Tuan, C. C. Byung, T. L. Kwon, and T. J. Yeon, "A simple approach for immobilization of gold nanoparticles on graphene oxide sheets by covalent bonding," Applied Surface Science, vol. 257, no. 8, pp. 3350-3357, 2011.

[15] K. Jasuja and V. Berry, "Implantation and growth of dendritic gold nanostructures on graphene derivatives: electrical property tailoring and Raman enhancement," ACS Nano, vol. 3, no. 8, pp. 2358-2366, 2009.

[16] Y. K. Kim, H. K. Na, and D. H. Min, "Influence of surface functionalization on the growth of gold nanostructures on graphene thin films," Langmuir, vol. 26, no. 16, pp. 1306513070, 2010.

[17] T. A. Pham, S. M. Son, and Y. T. Jeong, "Water-dispersible multi-walled carbon nanotubes and novel hybrid nanostructures," Synthesis and Reactivity in Inorganic, Metal-Organic and Nano-Metal Chemistry, vol. 40, no. 4, pp. 216-224, 2010.

[18] D. Gingery and P. Bühlmann, "Formation of gold nanoparticles on multiwalled carbon nanotubes by thermal evaporation," Carbon, vol. 46, no. 14, pp. 1966-1972, 2008.

[19] A. Tello, G. Cardenas, P. Häberle, and R. A. Segura, "The synthesis of hybrid nanostructures of gold nanoparticles and carbon nanotubes and their transformation to solid carbon nanorods," Carbon, vol. 46, no. 6, pp. 884-889, 2008.

[20] S. Y. Moon, T. Kusunose, S. I. Tanaka, and T. Sekino, "Easy synthesis of a nanostructured hybrid array consisting of gold nanoparticles and carbon nanotubes," Carbon, vol. 47, no. 12, pp. 2924-2932, 2009.

[21] R. Zanella, E. V. Basiuk, P. Santiago et al., "Deposition of gold nanoparticles onto thiol-functionalized multiwalled carbon nanotubes," Journal of Physical Chemistry B, vol. 109, no. 34, pp. 16290-16295, 2005.

[22] A. Velamakanni, C. W. Magnuson, K. J. Ganesh et al., "Sitespecific deposition of $\mathrm{Au}$ nanoparticles in CNT films by chemical bonding," ACS Nano, vol. 4, no. 1, pp. 540-546, 2010.

[23] T. G. Kim, D. Ragupathy, A. I. Gopalan, and K. P. Lee, "Electrospun carbon nanotubes-gold nanoparticles embedded nanowebs: prosperous multi-functional nanomaterials," Nanotechnology, vol. 21, no. 13, Article ID 134021, 2010.

[24] C. Gomez-Navarro, R. T. Weitz, A. M. Bittner et al., "Electronic transport properties of individual chemically reduced graphene oxide sheets," Nano Letters, vol. 7, no. 11, pp. 34993503, 2007.

[25] A. Lerf, H. He, M. Forster, and J. Klinowski, "Structure of graphite oxide revisited," Journal of Physical Chemistry B, vol. 102, no. 23, pp. 4477-4482, 1998.

[26] X. Zhou, X. Huang, X. Qi et al., "In situ synthesis of metal nanoparticles on single-layer graphene oxide and reduced graphene oxide surfaces," Journal of Physical Chemistry C, vol. 113, no. 25, pp. 10842-10846, 2009. 

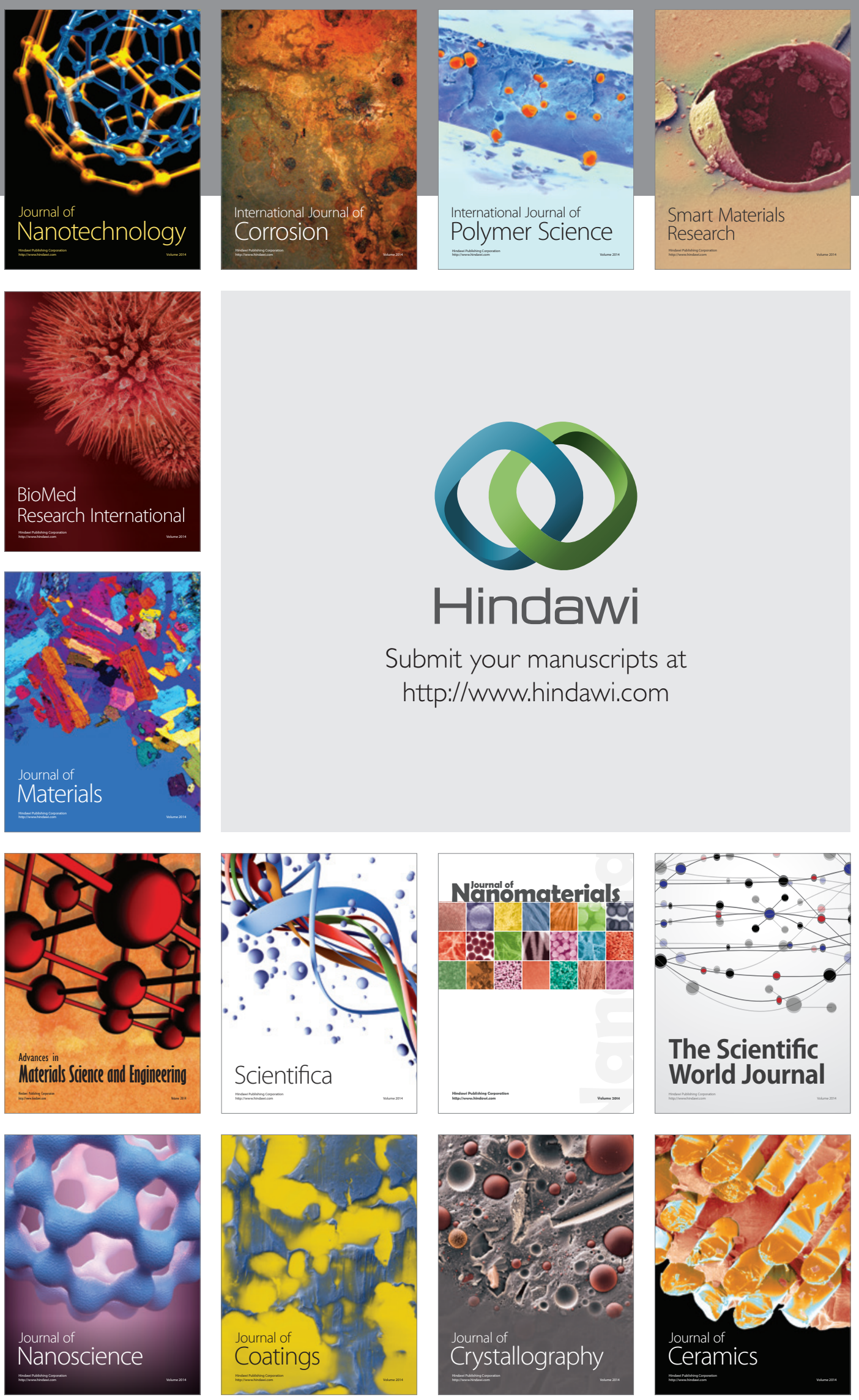

The Scientific World Journal

Submit your manuscripts at

http://www.hindawi.com

\section{World Journal}

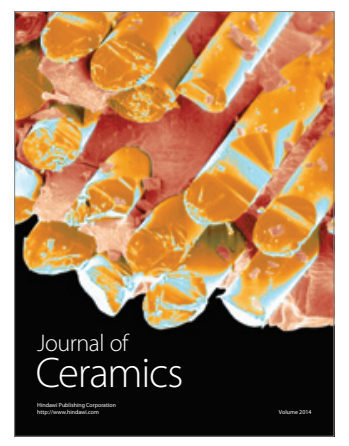

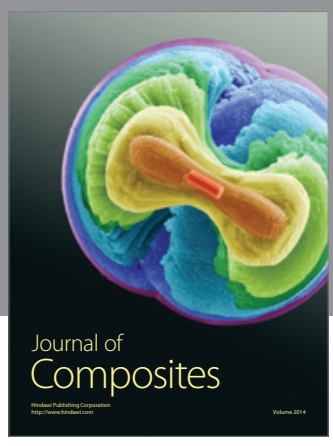
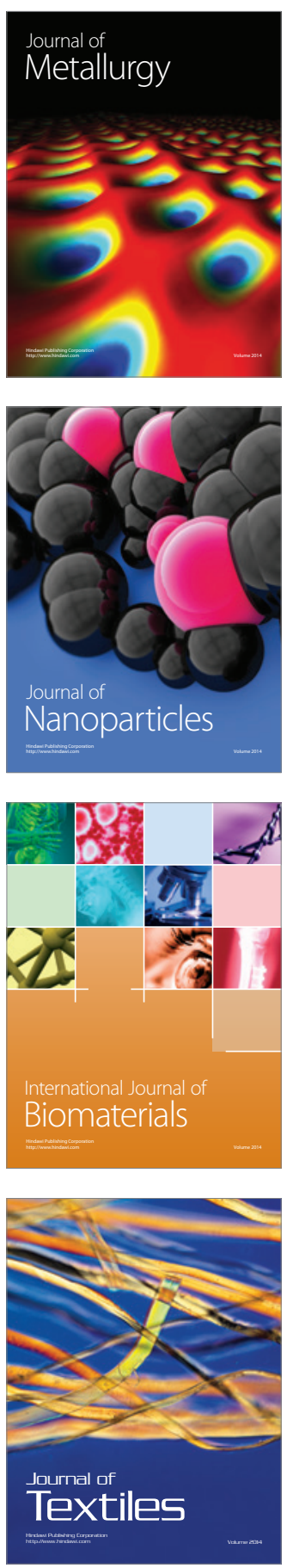